\title{
openheart Prevalence of cardiotropic viruses in adults with clinically suspected myocarditis in South Africa
}

\author{
Karim Hassan (D , , ${ }^{1}$ Charles Kyriakakis, ${ }^{1}$ Anton Doubell, ${ }^{1}$ Gert Van Zyl, ${ }^{2}$ \\ Mathilda Claassen, ${ }^{2}$ Dan Zaharie, ${ }^{3}$ Philip Herbst ${ }^{1}$
}

To cite: Hassan K, Kyriakakis C, Doubell A, et al. Prevalence of cardiotropic viruses in adults with clinically suspected myocarditis in South Africa. Open Heart 2022;9:e001942. doi:10.1136/ openhrt-2021-001942

Received 17 December 2021 Accepted 4 January 2022
D) Check for updates

\section{(C) Author(s) (or their} employer(s)) 2022. Re-use permitted under CC BY-NC. No commercial re-use. See rights and permissions. Published by BMJ.

${ }^{1}$ Division of Cardiology, Stellenbosch University Faculty of Medicine and Health Sciences, Cape Town, Western Cape, South Africa

'Division of Medical Virology, National Health Laboratory Service, Cape Town, Western Cape, South Africa

${ }^{3}$ Division of Anatomical Pathology, National Health Laboratory Service, Cape Town, Western Cape, South Africa

Correspondence to Dr Karim Hassan; hsskar@ gmail.com

\section{ABSTRACT}

Objectives To determine the prevalence and types of viral pathogens in the myocardium of patients presenting with clinically suspected myocarditis in South Africa.

Method This is a prospective cross-sectional study. Consecutive adults presenting to a single tertiary centre in South Africa between August 2017 and January 2021 who fulfilled the European Society of Cardiology's diagnostic criteria for clinically suspected myocarditis and who had undergone the appropriate investigations, including cardiac MRI (CMR) and endomyocardial biopsy (EMB), were included.

Results One hundred and two patients with clinically suspected myocarditis were enrolled. Acute myocarditis (AM) was confirmed by CMR or EMB in $82(80.39 \%)$ patients. Viral genomes were detected by PCR in EMB specimens of 50 patients with AM. Parvovirus B19 (PVB19) was the most frequently detected virus, in 37 as monoinfection and 4 as coinfection. This was followed by Epstein-Barr virus $(n=6)$, human herpesvirus $6(n=2)$ and human bocavirus $(n=1)$. PVB19 was also detected in 9 patients with no evidence of AM on CMR or EMB.

Conclusion Viral myocarditis is the most common form of myocarditis in South Africa. Local viral prevalence appears to be similar those of the developed world. The clinical significance and pathogenic role of PVB19 remains questioned, and its local background prevalence will have to be further investigated.

\section{INTRODUCTION}

Myocarditis is defined as an inflammatory disease of the heart muscle, diagnosed by established histological, immunological and immunohistochemical (IHC) criteria. ${ }^{1}$ Viral infections are the the most common cause of myocarditis in North America and Europe. ${ }^{1-4}$ The most common aetiology of myocarditis in Africa, and particularly South Africa, is unknown.

The developed world has experienced a shift in causative viral pathogens isolated from patients with myocarditis in recent years. Enteroviruses, including coxsackie virus, were the most prevalent between the 1950 s and 1990s, followed by adenovirus in

\section{Key questions}

What is already known about this subject?

- Viral infections are the most common cause of acute myocarditis (AM) in North America and Europe. The developed world has experienced a shift in viral pathogens detected on endomyocardial biopsy (EMB) specimens of patients with AM over the past 20 years, with parvovirus B19 (PVB19) and human herpesvirus 6 the most frequently detected viruses at present. The most common aetiology of $A M$ and the prevalence of viral pathogens in South Africa, and Africa as a whole, is unknown.

What does this study add?

- In this first study to investigate the prevalence of cardiotropic viruses in the myocardium of adults with clinically suspected AM in South Africa, we found that similar to data previously published from the developed world, viral infections are the most common cause of AM locally. Further, PVB19 $(82 \%)$ is the most frequently detected virus in the EMB specimens of patients with viral myocarditis. However, PVB19 was also detected in EMB specimens of patients without confirmed AM, raising the question of its clinical relevance and pathogenic role in viral myocarditis.

How might this impact on clinical practice?

- Viral myocarditis is the the most common form of myocarditis in both the developed and developing world. Further research needs to be conducted to investigate the clinical relevance and pathogenic role of PVB19 in myocarditis to guide clinical management of these patients.

the late 1990s. ${ }^{1-5}$ Parvovirus B19 (PVB19) and human herpes virus-6 (HHV6) have been increasingly detected in endomyocardial biopsy (EMB) of patients with acute myocarditis over the past 20 years, and these are now the most commonly identified viruses in patients with viral myocarditis. ${ }^{1-6}$ The reason behind this shift is unclear but is thought to be related to the routine evaluation of a broader repertoire of viruses, along with regional climate differences influencing the 
seasonal variation of viral infections. ${ }^{3}$ The aetiological roles and clinical significance of PVB19 and HHV6 in myocarditis, however, remain debated.

The viral prevalence in patients with myocarditis in both South Africa and Africa as a whole is also unknown. A recent South African study found that Epstein-Barr virus (EBV) was the most common virus detected on EMB specimens of patients with HIV associated cardiomyopathy, enterovirus the most common in patients with idiopathic dilated cardiomyopathy, and both enterovirus and adenovirus were the most frequently detected in heart transplant recipients. ${ }^{7}$ PVB19 was relatively uncommon and only isolated in 12\%-18\% of these patient groups. ${ }^{7}$ However, in this cohort, acute myocarditis was present in only $21 \%$ of patients with HIV-associated cardiomyopathy, $18 \%$ of heart transplant recipients, and none in those with idiopathic dilated cardiomyopathy.

This prospective study aims to determine the prevalence and types of viral pathogens in the EMB of patients presenting with clinically suspected myocarditis in a tertiary centre in Cape Town, South Africa.

\section{METHODS}

\section{Population and study design}

This is a single-centre prospective cross-sectional study. Consecutive patients over the age of 18 years presenting to Tygerberg Hospital, Cape Town, South Africa between August 2017 and January 2021 who fulfilled the European Society of Cardiology's (ESC) diagnostic criteria for clinically suspected myocarditis were enrolled. All potential patients underwent the full panel of recommended investigations, including cardiac MRI (CMR) and EMB. ${ }^{1}$ In brief, myocarditis was clinically suspected if patients presented with symptoms compatible with myocarditis, such as chest pain or symptoms of heart failure, accompanied by at least one additional finding on investigations supporting the diagnosis of myocarditis. This includes newly developed electrocardiographic (ECG) changes such as ST-T wave changes, atrioventricular block or ventricular tachyarrhythmias, evidence of cardiomyocyte necrosis in the form of elevated cardiac troponins, global or regional dysfunction of the left or right ventricle on transthoracic echocardiography (TTE) or CMR, and findings of oedema or a typical pattern of late gadolinium enhancement (LGE) on CMR. Myocarditis was also considered in patients who present with symptoms in keeping with an acute coronary syndrome without evidence of obstructive epicardial coronary artery disease or recent plaque rupture on coronary angiography.

All patients underwent a full clinical evaluation. Routine laboratory studies were performed which included a full blood count, renal function, high sensitivity troponin $\mathrm{T}$ (hsTnT) and $\mathrm{C}$ reactive protein (CRP). Additional laboratory studies were requested at the discretion of the attending physician. All patients also underwent a standard 12-lead ECG, TTE and CMR per standardised protocols described below. Coronary angiography was performed to exclude any significant epicardial coronary artery disease, defined as $\geq 50 \%$ stenosis in a single coronary artery segment. Right ventricular EMB was performed on all patients.

\section{Transthoracic echocardiography}

Comprehensive functional and structural 2-dimensional TTE were performed on all patients with Vivid S7 or Vivid E95 (GE Healthcare, Chicago, USA). Measurements were performed in accordance with the British Society of Echocardiography guidelines. ${ }^{8}$

\section{Cardiac MRI}

This was done in accordance with recommendations as set out in the Journal of the American College of Cardiology's white paper on CMR in myocarditis and 2018 update of CMR criteria for myocardial inflammation, as well as the Journal of Cardiovascular Magnetic Resonance's 2013 CMR protocol update. ${ }^{9-11}$ All imaging was done at the Tygerberg Hospital using a 1.5T field strength magnet (Magnetom Avanto; Siemens Healthcare GmbH, Erlangen, Germany). CMR analysis was carried out using commercially available software (CMR42, Circle CVI, Calgary, Canada).

In short: Standard long axis-axis and a short-axis stack of breath-held, retrospectively gated, steady-state freeprecession (SSFP) cine images were obtained. Postprocessing and image analysis were carried out using commercially available software (CMR42, Circle CVI, Calgary, Canada). Endocardial and epicardial left ventricular (LV) borders were traced in short axis at end-diastole and end-systole to determine LV volume, mass and functional parameters. Papillary muscles were excluded from the blood pool. Quantitative analysis of Short Tau Inversion Recovery images was performed following region of interest (ROI) contouring in short axis at basal, mid and apical LV level. A skeletal muscle (serratus anterior) ROI was manually drawn in the same slice for calculation of myocardial to skeletal muscle signal intensity ratios (SIR). Precontrast native T1 mapping images were obtained using a shortened Modified Look-Locker Inversion sequence. Standard T2-mapping was performed using a balanced SSFP readout sequence preceded by a multinomial T2-preparation module (Siemens Aera 1.5T). Early and LGE images were obtained with a T1-weighted, segmented, inversion recovery sequence performed at least $10 \mathrm{~min}$ after contrast administration. We used a standardised 16-segment model of the LV as outlined by the American Heart Association for regional assessment and to describe abnormalities. ${ }^{12}$ A gadolinium based contrast agent (Gadovist, Bayer AG, Leverkeusen, Germany) was administered at a standard cardiac dose of $0.2 \mathrm{~mL} / \mathrm{kg}$. In patients with an estimated glomerular filtration of less than $30 \mathrm{~mL} / \mathrm{min} / 1.732 \mathrm{~m}^{2}$, precluding the use of gadolinium-based contrast, native $\mathrm{T} 1$ time was used for assessing myocardial fibrosis.

CMR case definitions of acute myocarditis and CMR parameter analysis were based on the Lake Louise criteria. $^{9-11}$ 


\section{Endomyocardial biopsy}

Right ventricular septal biopsies were performed on all patients via femoral venous access with a modified Cordis bioptome (High Tech Medical, Johannesburg, South Africa) advanced through a 7 French Extra Back-Up (EBU) V.3.0 guiding catheter (Medtronic, Minneapolis, USA) under real-time fluoroscopic and TTE guidance. At least six specimens were taken from different sections of the septum to improve sensitivity. Three to four specimens were fixed in $4 \%$ buffered formalin for histological and IHC analysis, while the remaining samples were transported in $0.9 \%$ saline for viral genome detection by PCR.

\section{Histopathological and IHC analysis}

Specimens were assessed by a single anatomical pathologist at the National Health Laboratory Services. Light microscopy was performed on H\&E-stained slides, along with IHC testing using anti-CD3 (T lymphocytes), antiCD163 (macrophages) and anti-HLA-DR to define the types of immune cells. Additional stains such as Congo red was performed at the discretion of the pathologist. Myocarditis was diagnosed by either the Dallas histological criteria or the WHO/International Society and Federation of Cardiology (ISFC) IHCcriteria. ${ }^{13} 14$ Electron microscopy was performed on specimens fixed in $2 \%$ glutaraldehyde.

\section{Virological testing of samples}

To investigate viruses associated with myocarditis, a combination of multiplex and singleplex PCR assays were used. EMB material was split into two, for nucleic acid extraction, respectively with the Qiagen RNA easy Mini kit, for RNA extraction, and QIAamp DNA Mini kit (Qiagen, Hilden, Germany), for DNA extraction.

To detect Adenoviruses and Influenza viruses DNA and RNA was tested with the Anylplex ${ }^{\mathrm{T}}$ RV16 assay (Seegene, Seoul, South Korea); this assay also included parainfluenza viruses, human coronaviruses (229E, NL63 and OC43), human enteroviruses, respiratory syncytial viruses and human bocavirus. Herpesviruses, including human herpesvirus 1, 2, human cytomegalovirus, EBV and HHV6, were tested for with the Seeplex Meningitis-V1 ACE Detection assay (Seegene) from the DNA extract. In addition, DNA was tested with the PVB19 R-gene assay (bioMérieux, Marcy-l'Étoile, France) and to increase the sensitivity for human enteroviruses RNA was tested using a homebrew assay as previously described. ${ }^{15}$

\section{Definition of myocarditis}

For the purpose of the current study, myocarditis was diagnosed if either the original or updated LLC was met on CMR, or the Dallas histological criteria or WHO/ISFC IHC criteria fulfilled on EMB. ${ }^{9-11} 1314$

\section{Statistical analysis}

Normality of data was determined using the KolmogorovSmirnov test. Continuous variables were expressed as

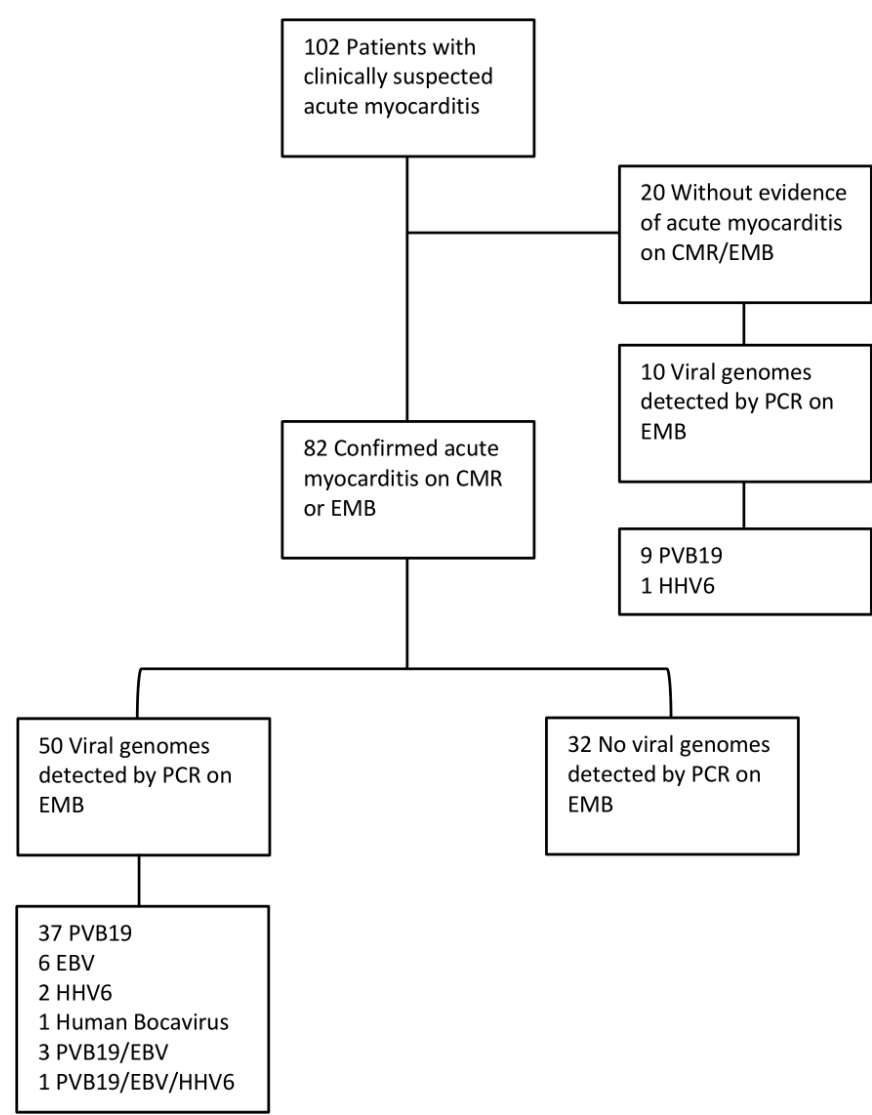

Figure 1 Patients enrolment. CMR, cardiac MRI; EMB, endomyocardial biopsy; HHV6, human herpesvirus 6 .

absolute numbers with associated percentages, mean and SD if normally distributed, or median and IQR if not normally distributed. Categorial variables were expressed as absolute numbers and percentages. Comparisons between groups were done by the use of Kruskal-Wallis test for non-normally distributed continuous variables and Student's t-test for normally distributed variables. The $\chi^{2}$ and Fisher's exact test were used for comparison of categorical variables. A two-tailed $\mathrm{p}<0.05$ was considered statistically significant.

\section{Patient and public involvement}

It was not possible to involve patients or the public in the design, conduct, reporting or dissemination of plans of this research study.

\section{RESULTS}

Between August 2017 and January 2021, 102 patients who presented to Tygerberg Hospital fulfilled the ESC diagnostic criteria for clinically suspected myocarditis (figure 1).

Acute myocarditis was confirmed in 82 patients: 40 (48.78\%) on CMR only, 15 (18.29\%) on EMB only and $27(32.93 \%)$ on both CMR and EMB. The baseline demographic data of patients with confirmed acute myocarditis and those without acute myocarditis are summarised and compared in table 1. Patients with confirmed myocarditis were significantly younger and had significantly higher 


\begin{tabular}{|c|c|c|c|}
\hline & Confirmed myocarditis $(n=82)$ & No myocarditis $(n=20)$ & $P$ value \\
\hline \multicolumn{4}{|l|}{ Demographics } \\
\hline Age (year) & $40.98 \pm 13.15$ & $47.25 \pm 11.66$ & 0.022 \\
\hline Sex, male $(\mathrm{n}, \%)$ & $54(65.85)$ & $12(60.00)$ & 0.623 \\
\hline HIV+ $(\mathrm{n}, \%)$ & $17(20.73)$ & $1(5.00)$ & 0.098 \\
\hline \multicolumn{4}{|c|}{ Laboratory investigations } \\
\hline White cell count & 7.91 (IQR 6.93-11.73) & 9.94 (IQR 7.46-13.13) & 0.4 \\
\hline $\mathrm{CRP}(\mathrm{mg} / \mathrm{L})$ & 17.00 (IQR 6.00-61.50) & 6.00 (IQR 3.00-13.00) & 0.044 \\
\hline hsTnT (ng/L) & 375.50 (IQR 61.75-990.75) & 40.50 (IQR 10.00-100.75) & 0.002 \\
\hline \multicolumn{4}{|c|}{ Echocardiographic measurements } \\
\hline LVEF (\%) & $39.98 \pm 13.91$ & $33.15 \pm 11.87$ & 0.103 \\
\hline $\operatorname{LVEDD}(\mathrm{mm})$ & $51.14 \pm 6.63$ & $57.00 \pm 6.48$ & 0.026 \\
\hline
\end{tabular}

CRP, C reactive protein; hsTnT, high sensitivity troponin T; LVEDD, left ventricular end diastolic diameter; LVEF, left ventricular ejection fraction.

median CRP and hsTnT at presentation. Those without myocarditis had significantly larger left ventricles on TTE.

Viral genomes were detected by PCR in $50(60.98 \%)$ patients with confirmed acute myocarditis. The prevalence of viral genomes detected in patients with confirmed myocarditis and without myocarditis are summarised in table 2.

\section{Patient characteristics}

The mean age of the cohort of virus-positive patients was 43.14 years. Thirty-one patients were male. Eleven patients were HIV positive with a mean CD4 count of 262. The findings of laboratory investigations, TTE, CMR and EMB are summarised in table 3.

There were no statistically significant differences between the mean age, median CRP and hsTnT at presentation, or mean LV ejection fraction (LVEF) and LV end diastolic diameter (LVEDD) between the virus-positive and virus-negative patients with confirmed acute myocarditis. However, those with viral myocarditis were significantly more likely to have evidence of acute myocarditis on EMB when compared with the virus-negative patients.

\begin{tabular}{lll}
\hline Table 2 & Viral genomes detected $(\mathrm{n}=60)$ \\
\hline & $\begin{array}{l}\text { With myocarditis } \\
(\mathbf{n}=50)\end{array}$ & $\begin{array}{l}\text { Without } \\
\text { myocarditis } \\
(\mathbf{n}=10)\end{array}$ \\
\hline Virus (n, \%) & & \\
\hline Parvovirus B19 (PVB19) & $37(74)$ & $9(90)$ \\
\hline Epstein-Barr virus (EBV) & $6(10)$ & $0(0)$ \\
Human herpesvirus 6 (HHV6) & $2(4)$ & $1(10)$ \\
Human bocavirus & $1(2)$ & $0(0)$ \\
Enterovirus & $0(0)$ & $0(0)$ \\
Adenovirus & $0(0)$ & $0(0)$ \\
PVB19/EBV & $3(6)$ & $0(0)$ \\
PVB19/EBV/HHV6 & $1(2)$ & $0(0)$ \\
\hline
\end{tabular}

\section{Parvovirus B19}

Forty-one patients with confirmed myocarditis had PVB19 detected on EMB either as monoinfection (37) or coinfection (4). Nine patients with PVB19 detected on EMB had no evidence of myocarditis on CMR or EMB (table 4).

There were no statistically significant differences between the mean age, mean LVEF, mean LVEDD, or the median CRP between those with and without confirmed myocarditis at presentation. Those with confirmed myocarditis however, had statistically significantly higher hsTnT at presentation when compared with those without.

\section{DISCUSSION}

The findings of this study confirm that viral myocarditis is the most common form of acute myocarditis in an African population presenting with clinically suspected myocarditis. The range of viral pathogens isolated on EMB of patients with suspected myocarditis at our centre is similar to those reported in North America and Europe. ${ }^{1-4}$

Viral infections have been previously reported as the most common cause of myocarditis in North America and Europe. ${ }^{1-5}$ The findings of our study show that viral myocarditis also appears to be the most common form of myocarditis locally, with viral genomes isolated in EMB specimens of $60.98 \%$ of 82 patients with confirmed acute myocarditis.

Although enteroviruses and adenoviruses were considered the most important causes of viral myocarditis in the $1950 \mathrm{~s}-1990 \mathrm{~s}$, there had been a shift in viral pathogens identified over the past 20 years, with PVB19 and HHV6 emerging as the most prevalent viruses isolated. ${ }^{1-6}$ The reason behind this shift remains debated and might involve geographical and temporal epidemiological differences in viral infections, or advances in diagnostic techniques, such as the routine use of PCR and in situ hybridisation, leading to the detection of a much broader 
Table 3 Baseline characteristics of patients with confirmed acute myocarditis $(n=82)$

\begin{tabular}{|c|c|c|c|}
\hline & Virus-positive $(n=50)$ & Virus-negative $(n=32)$ & $P$ value \\
\hline \multicolumn{4}{|l|}{ Demographics } \\
\hline Age (year) & $43.14 \pm 13.39$ & $37.59 \pm 12.20$ & 0.362 \\
\hline Sex, male $(n, \%)$ & $31(62.00)$ & $23(71.86)$ & 0.358 \\
\hline $\mathrm{HIV}+(\mathrm{n}, \%)$ & $11(22.00)$ & $6(18.75)$ & 0.723 \\
\hline CD4 (cells/ $/$ l) & $262 \pm 192$ & $354 \pm 182$ & 0.429 \\
\hline \multicolumn{4}{|c|}{ Laboratory investigations } \\
\hline White cell count & 10.36 (IQR 8.09-13.13) & 9.29 (IQR 7.28-13.01) & 0.96 \\
\hline $\mathrm{CRP}(\mathrm{mg} / \mathrm{L})$ & 24.00 (IQR 5.50-71.50) & 16.00 (IQR 6.50-35.00) & 0.241 \\
\hline hsTnT (ng/L) & 326.50 (IQR 46.50-1026.75) & 434.50 (IQR 131.00-925.50) & 0.739 \\
\hline \multicolumn{4}{|c|}{ Echocardiographic measurements } \\
\hline LVEF (\%) & $39.58 \pm 13.83$ & $40.61 \pm 14.24$ & 0.972 \\
\hline $\operatorname{LVEDD}(\mathrm{mm})$ & $50.74 \pm 6.70$ & $51.77 \pm 6.56$ & 0.285 \\
\hline \multicolumn{4}{|c|}{ CMR (Lake Louise Criteria) } \\
\hline Positive (n, \%) & $39(78.00)$ & $28(87.50)$ & 0.278 \\
\hline \multicolumn{4}{|c|}{ EMB (Dallas/IHC Criteria) } \\
\hline Positive (n, \%) & $30(60.00)$ & $12(37.50)$ & 0.03 \\
\hline
\end{tabular}

CMR, cardiac MRI; CRP, C reactive protein; EMB, endomyocardial biopsy ; hsTnT, high sensitivity troponin T; LVEDD, left ventricular end diastolic diameter; LVEF, left ventricular ejection fraction.

range of cardiotropic viruses. ${ }^{3}$ Similar to recent studies in the developed world, PVB19 appears to be the predominant viral pathogen in our setting, having been isolated in 41 out of 50 patients with viral myocarditis $(82 \%)$ as both monoinfection $(n=37)$ or coinfection $(n=4)$. It was surprising that neither enteroviruses nor adenoviruses were detected in our cohort of 102 patients with clinically suspected myocarditis. Whether this truly represents a shift in viral pathogens leading to myocarditis locally is unclear, as there are no previous studies that assessed viral prevalence in a similar patient population.

Our findings are in contrast to those of the only other local study to assess the viral prevalence on EMB specimens. In a small cohort of patients with HIV-associated cardiomyopathy $(\mathrm{n}=14)$, idiopathic dilated cardiomyopathy $(n=8)$ and heart transplant recipients $(n=11)$; EBV $(64 \%)$, enterovirus $(56 \%)$ and both enterovirus $(50 \%)$ and adenovirus $(50 \%)$ were found to be the most prevalent viruses respectively. ${ }^{7}$ PVB19 was only detected in $12 \%-18 \%$ of patients. ${ }^{7}$ There was evidence of myocarditis in $42.8 \%$ of patients with HIV-associated cardiomyopathy, $25 \%$ of idiopathic dilated cardiomyopathy and $36 \%$ of heart transplant recipients. ${ }^{7}$ The findings of this study and our study are, however, not directly comparable due to differences in sample sizes and population studied.

Interestingly, a single case of human bocavirus myocarditis was diagnosed in our study. Human bocavirus is a member of the Parvoviridae family first discovered in $2005 .^{16}$ It has been detected in individuals of all ages, although it predominantly affects infants of 6-24 months with respiratory tract disease or gastroenteritis. ${ }^{16}$ The detection of the virus in surgical myocardial specimens of healthy individuals indicates its cardiotropic features. ${ }^{17}$ Its pathogenicity is currently unknown, as it can only be cultured in differentiated human airway epithelial cells and there are no animal models available. ${ }^{16} 18$ To our knowledge, this is the first case of human bocavirus myocarditis confirmed both histologically and by viral PCR on EMB. Several cases of suspected human bocavirus myocarditis have been described, although most

Table 4 Comparison between patients with PVB19 isolated on EMB and evidence of acute myocarditis and those without evidence of acute myocarditis

\begin{tabular}{llll}
\hline & With myocarditis $(\mathbf{n}=\mathbf{4 1})$ & Without myocarditis $(\mathbf{n}=\mathbf{9})$ & P value \\
\hline Age (year) & $45.32 \pm 13.2$ & $41.89 \pm 9.36$ & 0.455 \\
LVEF (\%) & $39 \pm 14$ & $35 \pm 12$ & 0.455 \\
LVEDD (mm) & $51.17 \pm 6.87$ & $53.89 \pm 5.69$ & 0.176 \\
CRP (mg/L) & $22.00(6.00-72.75)$ & $4.50(2.50-50.75)$ & 0.138 \\
hsTnT (ng/L) & $295.00(46.00-985.50)$ & $50.00(10.00-110.00)$ & 0.011 \\
\hline
\end{tabular}

CRP, C reactive protein; EMB, endomyocardial biopsy; hsTnT, high sensitivity troponin T; LVEDD, left ventricular end diastolic diameter; LVEF, left ventricular ejection fraction; PVB19, parvovirus B19. 
of these were in children and only one adult case. ${ }^{16-22}$ Myocarditis was confirmed histologically in only two of these cases, while viral genome was detected in nasopharyngeal or oropharyngeal specimens or in blood, but not in the myocardium. ${ }^{18} 19$

The clinical relevance and causal role of PVB19 in myocarditis remains controversial and debated, as it had been detected in between $60 \%$ and $85 \%$ of postmortem subjects and individuals undergoing cardiac surgery without any evidence of myocarditis or cardiomyopathy in German cohorts. ${ }^{1722}{ }^{23}$ This suggests that PVB19 might be a mere bystander rather than causal agent in myocarditis. However, the background prevalence of PVB19 appears to vary depending on population studied, as it was detected in only $26 \%$ and $44 \%$ of postmortem cohorts without histological evidence of myocarditis in the United States and Denmark, respectively, and $44 \%$ of an Italian cohort undergoing cardiac surgery. ${ }^{24-26}$

Primary PVB19 infection usually occurs in childhood and manifests as erythema infectiosum. ${ }^{6} 2728$ Although infection in most individuals is transient, there is evidence to suggest lifelong persistence of the virus in certain tissue types including liver, synovium and skin. ${ }^{627} 28$ This is further supported by the demonstration of the presence of a genotype of PVB19 that had stopped circulating in Europe more than 50 years ago only in specimens obtained from patients born before $1973{ }^{28}$ Early studies into myocarditis and dilated cardiomyopathy showed a low prevalence of PVB19 in the myocardium of control subjects, supporting the hypothesis that PVB19 plays an important role in the pathogenesis of myocarditis and dilated cardiomyopathy. ${ }^{6}$ In contrast, more recent studies had shown a very high prevalence in hearts of patients without evidence of myocarditis, suggesting that similar to other tissue, PVB19 might also persist lifelong in the myocardium, and its mere presence might be insufficient to prove a direct causal role in disease.

It has been proposed that only the presence of high copy numbers of PVB19 DNA in the myocardium should be considered as significant and causative of myocarditis, with the current threshold determined to be more than 500 copies/ $\mu$ g DNA. ${ }^{329}$ There may, however, be geographical variations in this threshold, as a Dutch group determined by a postmortem study on hearts of patients without myocarditis that more than 250 copies/ $\mu \mathrm{g}$ DNA was significant for their population. ${ }^{30}$ Of the 50 patients with clinically suspected myocarditis who had PVB19 genomes detected on their EMB, nine had no evidence of myocardial inflammation on CMR or EMB. This supports the theory that PVB19 is a mere bystander rather than causal pathogen. Although there is no guideline approved therapy for PVB19-associated myocarditis and inflammatory cardiomyopathy, high dose IVIG has been shown in registry data and a single pilot to lead to significant clinical improvement, reduction in myocardial inflammation and improvement in LVEF in these patients. ${ }^{329}$ However, the current local cost of a single course of IVIG at the recommended dose is equivalent to 90 months' supply of heart failure therapy consisting of sacubitril/valsartan, dapagliflozin, carvedilol and spironolactone. It is thus important to further investigate the background prevalence of PVB19 and the viral load threshold for clinical significance in our resource-limited setting.

\section{Limitations}

This study was performed in a single centre and its results may not be generalisable to other populations. Although this likely represents the largest cohort of clinically suspected myocarditis patients who have all undergone $\mathrm{EMB}$, the sample size remains relatively small.

\section{CONCLUSION}

Viral myocarditis is the predominant form of myocarditis in our centre. The shift in viral pathogens witnessed in the developed world over the past 20 years, from enteroviruses and adenoviruses to PVB19, also appears to have taken place locally. The clinical relevance and pathogenic role of PVB19 in myocarditis in our local population remains questioned, as its presence was demonstrated in hearts of patients with and without myocarditis. Its background prevalence and the viral load threshold for clinical significance in the local population will need to be further investigated.

Contributors KH, CK, AFD, GVZ, DZ and PGH conceptualised the study. KH led the data collection with contribution from CK, GVZ, MC, DZ and PGH. KH led the data analysis and interpretation. KH wrote the first draft of the manuscript. All authors critically reviewed the manuscript and approved the final draft of the manuscript. All authors had full access to all the data in the study and had final responsibility for the decision to submit for publication. $\mathrm{KH}$ is the guarantor of the study and its content.

Funding The authors have not declared a specific grant for this research from any funding agency in the public, commercial or not-for-profit sectors.

Competing interests None declared.

Patient consent for publication Not applicable.

Ethics approval This study was approved by the Stellenbosch University Health Ethics Committee with the reference number of S20/10/273.

Provenance and peer review Not commissioned; externally peer reviewed.

Data availability statement Data are available on reasonable request.

Open access This is an open access article distributed in accordance with the Creative Commons Attribution Non Commercial (CC BY-NC 4.0) license, which permits others to distribute, remix, adapt, build upon this work non-commercially, and license their derivative works on different terms, provided the original work is properly cited, appropriate credit is given, any changes made indicated, and the use is non-commercial. See: http://creativecommons.org/licenses/by-nc/4.0/.

ORCID iD

Karim Hassan http://orcid.org/0000-0002-1141-9486

\section{REFERENCES}

1 Caforio ALP, Pankuweit S, Arbustini E, et al. Current state of knowledge on aetiology, diagnosis, management, and therapy of myocarditis: a position statement of the European Society of cardiology Working group on myocardial and pericardial diseases. Eur Heart J 2013;34:2636-48.

2 Buggey J, EIAmm CA. Myocarditis and cardiomyopathy. Curr Opin Cardiol 2018;33:341-6. 
3 Tschöpe C, Ammirati E, Bozkurt B, et al. Myocarditis and inflammatory cardiomyopathy: current evidence and future directions. Nat Rev Cardiol 2021;18:1-25.

4 Pollack A, Kontorovich AR, Fuster V, et al. Viral myocarditis-diagnosis, treatment options, and current controversies. Nat Rev Cardiol 2015;12:670-80.

5 Heymans S, Eriksson U, Lehtonen J, et al. The Quest for New Approaches in Myocarditis and Inflammatory Cardiomyopathy. J Am Coll Cardiol 2016;68:2348-64.

6 Verdonschot J, Hazebroek M, Merken J, et al. Relevance of cardiac parvovirus B19 in myocarditis and dilated cardiomyopathy: review of the literature. Eur J Heart Fail 2016;18:1430-41.

7 Shaboodien G, Maske C, Wainwright H, et al. Prevalence of myocarditis and cardiotropic virus infection in Africans with HIVassociated cardiomyopathy, idiopathic dilated cardiomyopathy and heart transplant recipients: a pilot study: cardiovascular topic. Cardiovasc J Afr 2013;24:218-23.

8 Masani N, Wharton G, Allen J. Echocardiography: Guidelines for Chamber Quantification British Society of Echocardiography Education Committee [Internet]. Available: https://www.bsecho.org/ media/40506/chamber-final-2011_2_.pdf

9 Friedrich MG, Sechtem U, Schulz-menger J, et al. Cardiovascular MRI in myocarditis. J Am Coll Cardiol 2009;53:1475-87.

10 Ferreira VM, Schulz-Menger J, Holmvang G, et al. Cardiovascular Magnetic Resonance in Nonischemic Myocardial Inflammation. J Am Coll Cardiol 2018;72:3158-76.

11 Kramer CM, Barkhausen J, Flamm SD, et al. Standardized cardiovascular magnetic resonance (CMR) protocols 2013 update. $J$ Cardiovasc Magn Reson 2013;15:91.

12 Cerqueira MD, Weissman NJ, Dilsizian V, et al. Standardized myocardial segmentation and nomenclature for tomographic imaging of the heart. A statement for healthcare professionals from the Cardiac Imaging Committee of the Council on Clinical Cardiology of the American Heart Association. Circulation 2002;105:539-42.

13 Aretz HT, Billingham ME, Edwards WD, et al. Myocarditis. A histopathologic definition and classification. Am J Cardiovasc Pathol 1987;1:3-14.

14 Richardson P, McKenna W, Bristow M, et al. Report of the 1995 World Health Organization/International Society and Federation of Cardiology Task Force on the Definition and Classification of Cardiomyopathies. Circulation 1996;93:841-2.

15 Watzinger F, Suda M, Preuner S, et al. Real-time quantitative PCR assays for detection and monitoring of pathogenic human viruses in immunosuppressed pediatric patients. J Clin Microbiol 2004;42:5189-98.

16 Guido M, Tumolo MR, Verri T, et al. Human bocavirus: current knowledge and future challenges. World J Gastroenterol 2016;22:8684-97.
17 Kuethe F, Lindner J, Matschke K, et al. Prevalence of parvovirus B19 and human bocavirus DNA in the heart of patients with no evidence of dilated cardiomyopathy or myocarditis. Clin Infect Dis 2009;49:1660-6.

18 Brebion A, Vanlieferinghen P, Déchelotte P, et al. Fatal subacute myocarditis associated with human bocavirus 2 in a 13-month-old child. J Clin Microbiol 2014:52:1006-8.

19 Sallmon H, Lopez E, Weber S, et al. Subacute myocarditis associated with bocavirus infection in an 8-week-old infant. Klin Padiatr 2017;229:103-5.

20 Heydari H, Mamishi S, Khotaei G-T, et al. Fatal type 7 adenovirus associated with human bocavirus infection in a healthy child. J Med Virol 2011;83:1762-3.

21 Carvalho F, Pessoa C, Rocha L. Viral myocarditis due to bocavirus: fact or mere coincidence? Crit Care Med 2013;41.

22 Schenk T, Enders M, Pollak S, et al. High prevalence of human parvovirus B19 DNA in myocardial autopsy samples from subjects without myocarditis or dilative cardiomyopathy. J Clin Microbiol 2009;47:106-10.

23 Lotze U, Egerer R, Glück B, et al. Low level myocardial parvovirus B19 persistence is a frequent finding in patients with heart disease but unrelated to ongoing myocardial injury. J Med Virol 2010;82:1449-57.

24 Koepsell SA, Anderson DR, Radio SJ. Parvovirus B19 is a bystander in adult myocarditis. Cardiovasc Pathol 2012;21:476-81.

25 Nielsen TS, Hansen J, Nielsen LP, et al. The presence of enterovirus, adenovirus, and parvovirus B19 in myocardial tissue samples from autopsies: an evaluation of their frequencies in deceased individuals with myocarditis and in non-inflamed control hearts. Forensic Sci Med Pathol 2014;10:344-50.

26 Moimas S, Zacchigna S, Merlo M, et al. Idiopathic dilated cardiomyopathy and persistent viral infection: lack of association in a controlled study using a quantitative assay. Heart Lung Circ 2012;21:787-93.

27 Corcoran C, Hardie D, Yeats J, et al. Genetic variants of human parvovirus B19 in South Africa: cocirculation of three genotypes and identification of a novel subtype of genotype 1. J Clin Microbiol 2010;48:137-42.

28 Norja P, Hokynar K, Aaltonen L-M, et al. Bioportfolio: lifelong persistence of variant and prototypic erythrovirus DNA genomes in human tissue. Proc Natl Acad Sci U S A 2006;103:7450-3.

29 Ammirati E, Frigerio M, Adler ED, et al. Management of acute myocarditis and chronic inflammatory cardiomyopathy: an expert consensus document. Circ Heart Fail 2020;13:e007405.

30 Dennert R, Velthuis S, Schalla S, et al. Intravenous immunoglobulin therapy for patients with idiopathic cardiomyopathy and endomyocardial biopsy-proven high PVB19 viral load. Antivir Ther 2010;15:193-201. 